\title{
Data Learning of Fluid Dynamics for Physically Informed Digital Twins
}

\author{
Beatriz Moya ${ }^{1}$, Icíar Alfaro ${ }^{1}$, David González ${ }^{1}$, Francisco Chinesta $^{2}$, Elías Cueto ${ }^{1}$ \\ ${ }^{1}$ Applied Mechanics and Bioengineering (AMB) \\ Instituto de Investigación en Ingeniería de Aragón (I3A) \\ Universidad de Zaragoza, Mariano Esquillor s/n, 50018, Zaragoza, Spain. \\ Tel. +34-976762707, e-mail: beam@unizar.es \\ ${ }^{2}$ ENSAM ParisTech
}

\begin{abstract}
We present a novel real-time digital twin based on artificial intelligence to emulate physically sound fluid dynamics, and classify and recognize liquids, with information from video streaming. Results are presented with augmented reality techniques not only for friendly user interaction, but also to provide augmented information in manipulation tasks.
\end{abstract}

\section{Introduction}

A so-called digital twin represents a copy of an object of process which is our object of study [1]. The main difference between a virtual simulation and a digital twin recalls in its connection with its physical twin. We obtain real-time data to feed the copy with the same stimulus than the real counterpart and output the same reaction. Digital twins are commonly present in industrial and engineering applications. Also, as we suggest in this work, they could set the robotics reasoning basis to translate real world information into a simulation and decision making engine.

In this work, we have built a digital twin that learns the behavior of a previously unknown fluid. It works in a closed loop where we relate the velocity of the glass with the sloshing results of the liquid. We show to the user by means of augmented reality a representation of a virtual copy of the fluid that accomplishes physical consistency of the results. The learning process is constrained by the laws of thermodynamics to provide interpretable results, with which we can perform informed and accurate decision making.

\section{Method}

We make use of different machine learning techniques to learn from data dynamics prediction and fluid recognition. These capabilities are pretrained in an offline phase with pseudo-experimental data obtained from computational simulations.
These simulations are performed under the assumption of the Smooth Particles Hydrodynamics discretization theory to simulate complex sloshing behaviors.

\section{Thermodynamically consistent simulation engine}

The work done in [2] has been extended to a wider selection of fluids to learn to emulate dynamics of both Newtonian and Non-Newtonian fluids. Due to the diversity and complexity of the behaviors, we learn a model for each fluid. We perform machine learning of 3D fluid dynamics under the restrictions imposed by the GENERIC formalism [3]. It stablishes a theoretical framework to describe the evolution of energy $(\boldsymbol{L} \partial E / \partial \boldsymbol{z})$ and entropy $(\boldsymbol{M} \partial S /$ $\partial z$ ) of the system in terms of and adequate selection of state variables $\mathbf{z}$, which must appropriately describe the time evolution of the dynamics

$$
\frac{d \mathbf{z}}{d t}=\boldsymbol{L} \frac{\partial E}{\partial \boldsymbol{z}}+\boldsymbol{M} \frac{\partial S}{\partial \boldsymbol{z}}
$$

By defining the Hamiltonian matrix $\mathbf{L}$ skewsymmetric, and the friction matrix $\mathbf{M}$ symmetric positive semidefinite, and accomplishing the degeneracy conditions, we ensure thermodynamic consistency

$$
\boldsymbol{L} \frac{\partial S}{\partial \boldsymbol{z}}=\mathbf{0}, \quad \boldsymbol{M} \frac{\partial E}{\partial \mathbf{z}} \geq \mathbf{0}
$$

Given the high dimensionality of the problem, it would be challenging to achieve real-time performance. We project the data available into a space, or manifold, of much lower dimensionality by means of k-PCA, where GENERIC conditions are also ensured. We reach an embedded space of 4 dimensions to carry out the learning process of discretized GENERIC. 


\section{Fluid recognition}

We also explore the clustering characteristics of the database by projecting data of all the fluids analyzed to a manifold of 3 dimensions with k-PCA. In this projection, one can appreciate that the fluids remain clustered with regard to its properties. With this result, we train a random forest classifier that we feed with new and material-unknown data to predict which fluid we manipulate.

\section{Data acquisition}

A critical point for a digital twin is its connection with the real world. Despite that sensors are a commonly used tool for data acquisition, the latest computer vision techniques allow fast and accurate information extraction from the environment. First, we need to feed the model with the velocity of the glass. We track points of interest, or features, of the pattern added to the transparent container of the fluid, to compute the position and velocity of the glass. In a second phase, we have developed a system to detect points of the free surface of the liquid we track and obtain information of the liquid (see Figure 1).

\section{Results}

The development of the twin has been performed obtaining promising results. Random forest classifier obtains a global accuracy of $95.93 \%$, precision of $99.17 \%$, and recall of $99.17 \%$, which are considered successful. The simulation model is implemented to work with a video streaming from a stereo camera. Both are coupled and at real-time frequency, and the result is presented in augmented reality, obtaining mean deviations of less than $2 \mathrm{~mm}$ (see Figure 2).

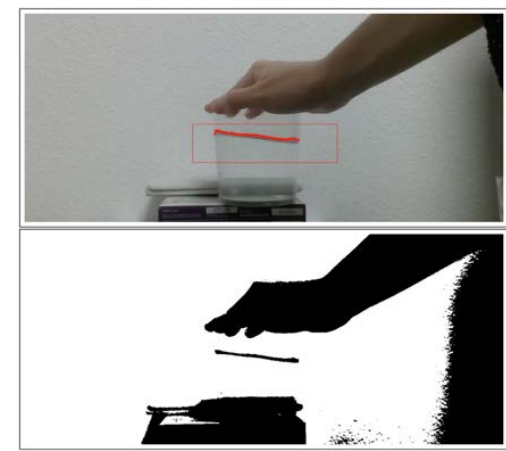

Fig. 1. Free surface detection for data acquisition. The video frame is binarized to detect and track points of the surface.

\section{Conclusions}

We have proved the efficacy of GENERIC to develop accurate and physically consistent digital twins. This fact, in conjunction with the data acquisition systems developed, and results of fluid classification, set the base for the development of a hybrid twins able to correct itself.

\section{Acknowledgements}

This work has been partially supported by the Spanish Ministry of Economy and Competitiveness through Grant number DPI2017-85139-C2-1-R and by the Regional Government of Aragon and the European Social Fund, research group T88. The support of ESI Group through the project UZ-20190060 is also gratefully acknowledged.

\section{REFERENCES}

[1]. CHINESTA, F., CUETO, E., ABISSETCHAVANNE, E., DUVAL, J. L., EL KHALDI, F. (2020). Virtual, digital and hybrid twins: a new paradigm in data-based engineering and engineered data. Archives of computational methods in engineering, 27(1), 105-134.

[2]. MOYA, B., GONZÁLEZ, D., ALFARO, I., CHINESTA, F., CUETO, E. (2019) Learning slosh dynamics by means of data. Computational Mechanics, 64(2), 511-523.

[3]. GRMELA, M., ÖTTINGER, H. C. (1997). Dynamics and thermodynamics of complex fluids. I. Development of a general formalism. Physical Review E, 56(6), 6620.

[4]. MOYA, B., ALFARO, I., GONZALEZ, D., CHINESTA, F., CUETO, E. (2020) Physically sound, self-learning digital twins for sloshing fluids. PLOS One, 15(6), e0234569.

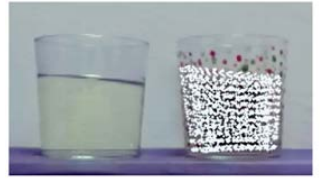

Snapshot 1-glycerine

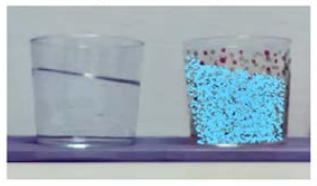

Snapshot 1-water

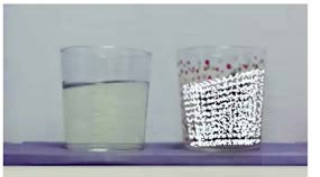

Snapshot 2-glycerine

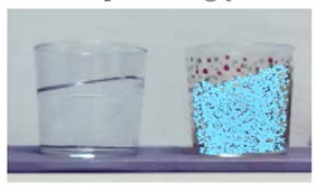

Snapshot 2-water
Fig. 2. Results of the digital twin implementation for glycerine and water. Two random snapshots have been selected from the full video sequence.

Revista “Jornada de Jóvenes Investigadores del I3A”, vol. 8 (Actas de la IX Jornada de Jóvenes Investigadores del I3A -11 de diciembre de 2020). ISSN 2341-4790. 